\title{
Caractériser la diversité des exploitations maraîchères de la région de Bobo-Dioulasso au Burkina Faso pour faciliter leur transition agroécologique
}

\author{
Rayangnéwendé Adèle Ouédraogo ${ }^{1,2, *}$, Fabèkourè Cédric Kambiré ${ }^{2}$, Marie-Paule Kestemont ${ }^{1}$ et \\ Charles L. Bielders ${ }^{1}$ \\ ${ }^{1}$ Université Catholique de Louvain, Croix du sud 2, L7.05.02, 1348 Louvain-la-Neuve, Belgique \\ 2 Centre National de Recherche Scientifique et Technologique 03, BP 7047, Ouagadougou 03, Burkina Faso
}

\begin{abstract}
Résumé - Une typologie basée sur les pratiques maraîchères a été réalisée à partir d'enquêtes auprès de 300 producteurs de dix périmètres maraîchers en milieu urbain, semi-urbain et rural de la région de BoboDioulasso au Burkina Faso, afin d'évaluer le potentiel d'évolution des exploitations vers des systèmes de culture plus écologiques. Aucun agriculteur ne pratique actuellement une production exclusivement biologique ou agroécologique. La typologie a permis de définir quatre types d'exploitations sur base de leur localisation, de leur superficie, de l'intensité d'utilisation des pesticides et des engrais minéraux et organiques. Ces exploitations présentent des atouts en commun, comme la pratique généralisée de la rotation et de l'association des cultures et de la fertilisation organique, mais aussi des défis spécifiques à relever en termes d'usage des pesticides de synthèse et de fertilisation raisonnée pour une transition agroécologique. Les producteurs ont généralement une connaissance faible et imprécise des effets de leurs pratiques sur l'environnement, tout particulièrement en milieu urbain où la sensibilisation aux impacts sanitaires et environnementaux des pratiques maraîchères doit être renforcée.
\end{abstract}

Mots clés : typologie / maraîchage / agroécologie / enquête / Burkina-Faso

\begin{abstract}
Characterizing the diversity of vegetable farms in the Bobo-Dioulasso region in Burkina Faso to facilitate their agro-ecological transition. A typology based on market gardening practices was carried out based on a survey of 300 producers from ten urban, semi-urban and rural market gardening sites of Bobo-Dioulasso in Burkina Faso, in view of assessing the potential for transition towards more ecological production methods. No farmer currently practices exclusively organic or agro-ecological production methods. The typology allowed defining four types of farms based on their location, size and intensity of use of pesticides and mineral and organic fertilizers. All farms have common assets such as the widespread practice of crop rotations and associations and organic fertilization, but also specific challenges in terms of pesticide use and sustainable fertilization for an agro-ecological transition. Producers generally have a weak and imprecise knowledge of the effects of their practices on the environment, especially in urban areas where greater awareness regarding the health and environmental impacts of market gardening practices needs to be raised.
\end{abstract}

Keywords: typology / vegetable gardens / agro-ecology / survey / Burkina-Faso

\section{Introduction}

De nos jours, l'agriculture en général, et le maraîchage en particulier, doivent faire face à de nombreux défis comme l'augmentation de l'offre alimentaire, mais aussi l'exigence

\footnotetext{
*Auteur de correspondance : charles.bielders@uclouvain.be
}

d'une production agricole garantissant la sécurité sanitaire, la qualité nutritionnelle et le respect de l'environnement. Cependant, dans de nombreux pays en développement, les réglementations en matière de techniques de production ou de qualité sanitaire des productions agricoles sont soit inexistantes, soit ne s'accompagnent pas d'un contrôle effectif des pratiques, des intrants et des produits alimentaires. Ainsi, la culture maraîchère en Afrique subsaharienne repose souvent 


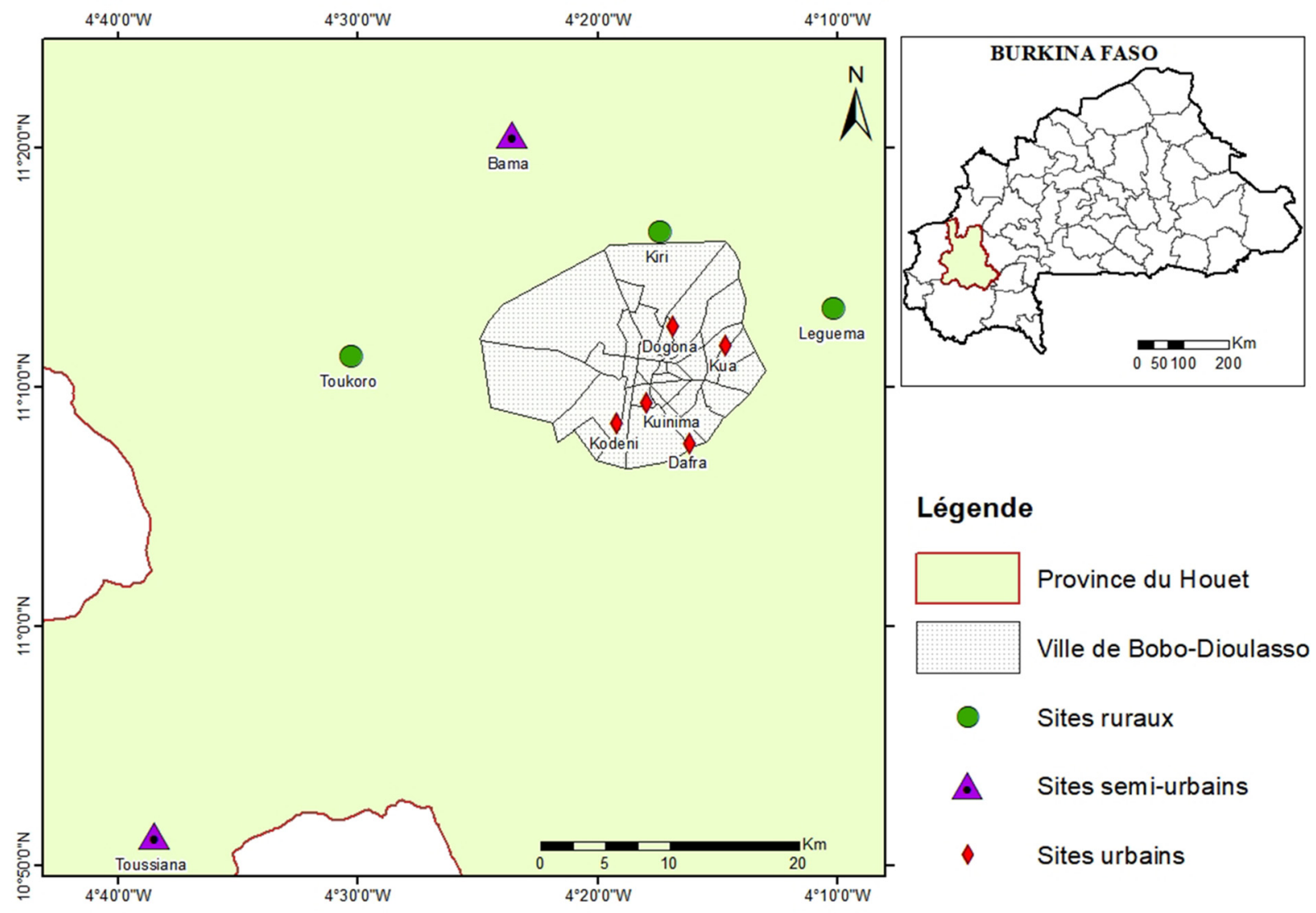

Fig. 1. Situation géographique des sites d'étude dans la province du Houet.

Fig. 1. Geographical location of study sites in the Houet province.

sur un usage intensif, voire abusif, d'intrants (engrais minéraux, déchets organiques, produits phytosanitaires, eaux usées), avec des conséquences souvent néfastes pour la santé humaine et l'environnement (Abdulkadir et al., 2013; Son et al., 2017).

Face à ces menaces, de plus en plus d'initiatives émergent afin de promouvoir des pratiques maraîchères plus respectueuses de la santé humaine et de l'environnement (Traoré et Toé, 2008), aussi bien en termes de fertilisation des cultures, de biocontrôle des maladies et ravageurs, de qualité des eaux d'irrigation, que de diversité des cultures. Ces initiatives s'inscrivent dans une démarche de promotion d'une production horticole raisonnée, préférablement biologique, voire agroécologique (dans sa signification technique; Stassart et al., 2012).

Dans ce travail, les sites maraîchers dans et aux alentours de la ville de Bobo Dioulasso au Burkina Faso sont utilisés comme cas d'étude en raison de la diversité des milieux de production (urbain, semi-urbain, rural) induisant des différences d'accès à l'eau et aux intrants, mais aussi d'accès à la terre ou aux marchés. Selon Gafsi et al. (2007), l'âge et le niveau d'instruction des exploitants, mais aussi le mode d'accès au foncier et aux ressources naturelles sont autant de facteurs à l'origine de la diversité des exploitations agricoles. Or, il est bien établi que la prise en compte de cette diversité est une condition essentielle d'amélioration de l'efficacité des interventions des acteurs de développement auprès des agriculteurs (Gafsi et al., 2007). Ainsi, afin de mieux guider les maraîchers vers une transition agroécologique, il paraît nécessaire dans un premier temps de caractériser, en fonction du milieu de production, les pratiques actuelles de production, de comprendre la logique qui guide le choix de ces pratiques, d'analyser la perception qu'ont les producteurs de l'impact de leurs pratiques et de déceler les freins à l'adoption de pratiques plus écologiques.

Compte tenu de la grande diversité des cultures et des pratiques maraîchères, et des nombreuses combinaisons possibles (Landais et al., 2016), l'élaboration de typologies apparaît comme un moyen de réduire la complexité tout en conservant une diversité pertinente par rapport aux objectifs visés. Bien que différentes typologies d'exploitations maraîchères aient été établies en Afrique de l'ouest (Dossa et al., 2011; Ahouangninou, 2013), celles-ci ne sont pas axées sur la caractérisation des pratiques maraîchères en lien avec les conditions de production. Ainsi, la réalisation d'une typologie des exploitations en fonction des pratiques observées pourra servir de base à la planification d'actions d'information, de formation et de vulgarisation de pratiques agroécologiques de la part des acteurs du développement.

\section{Matériels et méthodes}

L'étude est centrée sur Bobo-Dioulasso et sa périphérie, $2^{\mathrm{e}}$ ville du pays (Fig. 1), avec une pluviométrie annuelle 


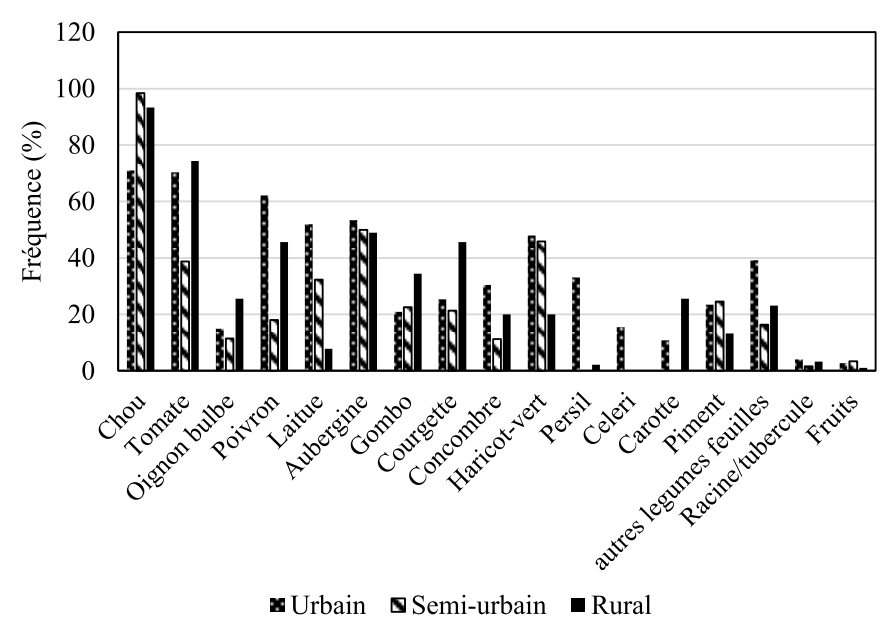

Fig. 2. Fréquence des cultures maraîchères (\% des exploitations) en fonction du type de périmètre maraîcher. Fruits: melon, fraises. Légumes feuilles : feuilles de patate, épinard, niébé, oignon, menthe, oseille, amarante. Racine/tubercule: pomme de terre, betterave, navet.

Fig. 2. Frequency of the crops produced (\% of farms) according to the type of market gardening area.

comprise entre 900 et $1200 \mathrm{~mm}$ (Robineau et Dugué, 2018). À partir d'une liste exhaustive des sites maraîchers dans un rayon de $50 \mathrm{~km}$ autour de la ville dont la vocation première est le maraîchage, 5 sites de production urbains (à l'intérieur de la ville: Kuinima, Kôdeni-Samagan, Dogona-Sakabi, Dafra et Kua), 2 sites semi-urbains (villes secondaires chefs-lieux de département comme Bama et Tousiana), et 3 sites ruraux (villages autour de la ville: Leguema, Toukoro et Kiri) ont été retenus pour cette étude (Fig. 1).

Pour chacun des 10 sites, 30 producteurs ont été interrogés. Les producteurs ont été choisis selon un échantillonnage systématique de sorte à couvrir au mieux les périmètres maraîchers retenus. Pour cela, un quadrillage a été superposé sur la carte de chaque périmètre. La taille des mailles a été choisie pour qu'il y ait exactement 30 intersections au sein du périmètre.

La collecte des données a été faite lors d'une enquête par questionnaire portant uniquement sur les activités maraîchères des 300 producteurs, d'observations directes de leurs pratiques et de mesures des superficies maraîchères au cours de la dernière année de production. L'enquête a porté sur une année complète de production et a concerné toutes les cultures maraîchères et les divers cycles de production (saison sèche ou contre saison, saison des pluies). Le questionnaire était structuré en trois parties: les caractéristiques générales de l'exploitation, les pratiques culturales et la perception qu'ont les producteurs de l'impact de leurs pratiques sur l'environnement et la qualité des productions.

Les doses d'intrants ont été estimées sur la base des quantités apportées et des superficies mesurées pour chaque culture maraîchère produite au cours de l'année de référence. Pour les engrais composés, la formulation NPK 15-15-15 la plus commune (fréquence $=64 \%$ ) a été utilisée comme référence pour les calculs. Pour la fumure organique, les teneurs en azote $(\mathrm{N})$, phosphore $(\mathrm{P})$ et potassium $(\mathrm{K})$ ont été déterminées sur 5 à 10 échantillons par type de fumure.
L'indicateur de fréquence de traitement phytosanitaire (IFT) a été utilisé pour pondérer les apports des différents pesticides.

Outre des analyses descriptives, une classification ascendante hiérarchique $(\mathrm{CAH})$ a été utilisée pour établir la typologie des maraîchers en fonction de leurs choix de pratiques culturales. En plus des variables discriminantes identifiées à l'issue de l'analyse descriptive (superficie exploitée, dose annuelle d'azote par ha, IFT, pourcentage d'azote organique dans les apports d'azote totaux, nombre moyen d'épandages de pesticides par culture), le milieu (urbain, semi-urbain ou rural), la source d'eau (puit/rivière) et le mode d'irrigation (arrosoir/pompe) ont été ajoutés comme variables supplémentaires. La pratique de l'association ou de la rotation culturale et l'utilisation de pesticides biologiques, l'âge et le niveau d'instruction des producteurs n'ont pas été retenus car ils ne permettaient pas de les discriminer suffisamment. Enfin, il a été demandé aux producteurs d'évaluer les impacts de leurs pratiques de fertilisation et d'utilisation de pesticides sur le sol, l'eau et les produits maraîchers et de proposer des alternatives techniques pour les limiter.

\section{Résultats}

\subsection{Caractéristiques sociodémographiques des maraîchers}

Selon les sites, 97 à $99 \%$ des maraîchers sont des hommes. Leur âge (médiane $=40$ ans) et expérience (médiane $=20$ ) ne diffèrent pas selon le type de milieu. Près de $60 \%$ des producteurs n'ont aucun niveau d'instruction et seulement 8 à $10 \%$ ont atteint le niveau secondaire. Les superficies exploitées en cultures maraîchères sur une année complète vont croissant du milieu urbain (médiane $=1259 \mathrm{~m}^{2}$ ), au milieu semi-urbain $\left(\right.$ médiane $\left.=2411 \mathrm{~m}^{2}\right)$ et rural $\left(\right.$ médiane $\left.=4957 \mathrm{~m}^{2}\right)$. En milieu urbain, semi-urbain et rural, respectivement 20 , 28 et $30 \%$ des producteurs ne sont pas propriétaires de leur parcelle.

\subsection{Pratiques culturales}

Les cultures dominantes, quel que soit le type de milieu, sont le chou $(88 \%)$, la tomate $(61 \%)$, l'aubergine $(51 \%)$, le poivron $(42 \%)$, le haricot vert $(38 \%)$ et la laitue $(31 \%)$. On observe cependant une plus grande diversité d'espèces cultivées en milieu urbain (26) comparée à celles des milieux semi-urbain (16) et rural (19) (Fig. 2). En moyenne, on recense 5 espèces différentes par producteur.

Dans les trois milieux, la rotation culturale est pratiquée par plus de $90 \%$ des producteurs. Les rotations les plus fréquentes en milieu urbain sont laitue-tomate $(11 \%)$, laitue-chou $(18 \%)$ et chou-tomate (11\%). En milieu semi-urbain : haricot vert-chou $(23 \%)$, chou-aubergine $(20 \%)$, laitue-chou $(15 \%)$ et choutomate $(12 \%)$. En milieu rural : chou-tomate $(23 \%)$, carottechou (14\%) et aubergine-chou ( $13 \%)$. Selon les producteurs, les rotations permettent de viser les périodes de forte rentabilité $(25 \%)$ tout en tenant compte des périodes favorables à chaque espèce $(55 \%)$. En général, les cultures à cycle court et moins exigeantes en intrants (laitue, haricot vert, amaranthe, oseille) viennent en tête de rotation (généralement en octobre), afin de 
Tableau 1. Fréquence des principaux types d'associations culturales en milieux urbain, semi-urbain et rural.

Table 1. Frequency of the main types of crop associations in urban, semi-urban and rural areas.

\begin{tabular}{lll}
\hline Milieu & Type d'association & Fréquence (\%) \\
\hline \multirow{4}{*}{ Urbain } & Poivron-laitue & 43 \\
& Tomate-laitue & 26 \\
& Laitue-autres légumes feuilles & 25 \\
& Chou-laitue & 16 \\
\multirow{4}{*}{ Semi-urbain } & Chou-laitue & 18 \\
& Tomate-maïs (faible densité) & 12 \\
& Tomate-laitue & 10 \\
& Tomate-gombo & 44 \\
Rural & Chou-gombo & 30 \\
& Tomate-légume feuille & 30 \\
& Tomate-maïs (faible densité) & 19 \\
\hline
\end{tabular}

générer rapidement des revenus pour l'achat des semences des principales cultures maraîchères de vente (poivron, chou, tomate), qui coûtent aussi plus cher en entretien du fait de la longueur de leur cycle et de leurs besoins en intrants (insecticide, engrais).

L'association culturale est pratiquée par 90,72 et $75 \%$ des producteurs urbains, semi-urbains et ruraux, respectivement. Les types d'association sont très diversifiés et les plus courants en milieu urbain sont: poivron-laitue $(43 \%)$, tomate-laitue $(26 \%)$, laitue-autre légume feuille $(25 \%)$ et chou-laitue $(16 \%)$. En milieu semi-urbain: chou-laitue $(18 \%)$, tomate comprenant du maïs à faible densité $(12 \%)$ et tomate-laitue $(10 \%)$. En milieu rural: tomate-gombo $(44 \%)$ ou chou-gombo (30\%), tomate-légume feuille (30\%) et tomate comprenant du maïs à faible densité (19\%) (Tab. 1). Selon les producteurs, les associations culturales, en plus de diversifier la production $(43 \%)$, permettent des rentrées financières plus régulières grâce aux récoltes étalées dans le temps, en particulier les légumes feuilles (92\%). Elles permettent aussi parfois de produire des légumes à des périodes moins favorables d'un point de vue climatique mais très intéressantes en termes de prix de vente. Par exemple, le poivron et la laitue se vendent bien en saison chaude, qui n'est cependant pas favorable à leur production. En les associant, la laitue conserve l'humidité du sol en réduisant l'évaporation (bénéfique pour le poivron) et en retour, le poivron crée de l'ombre pour la laitue, lui permettant de résister à la forte chaleur.

On note globalement des apports plus importants de nutriments $\mathrm{N}, \mathrm{P}, \mathrm{K}$ contenus dans les engrais minéraux et organiques en milieu urbain qu'en milieux semi-urbain et rural. Ainsi, les apports totaux de $\mathrm{N}$ sur les cultures maraîchères sur un cycle annuel de production représentent en moyenne $1385 \mathrm{~kg}$ $\mathrm{ha}^{-1} \mathrm{an}^{-1}$ en milieu urbain, contre 811 et $767 \mathrm{~kg} \mathrm{ha}^{-1} \mathrm{an}^{-1}$ en milieux semi-urbain et rural, respectivement (Fig. 3a). Les cultures les plus fertilisées sont le chou, la tomate, le poivron et le haricot vert, avec en moyenne respectivement $391,311,515$ et $267 \mathrm{~kg} \mathrm{~N} \mathrm{ha}^{-1}$ cycle $^{-1}$ (Fig. 3b).

La majorité des producteurs utilisent les amendements organiques, cette pratique étant plus présente en milieux urbain et semi-urbain (98\%) qu'en milieu rural $(83 \%)$. Six types
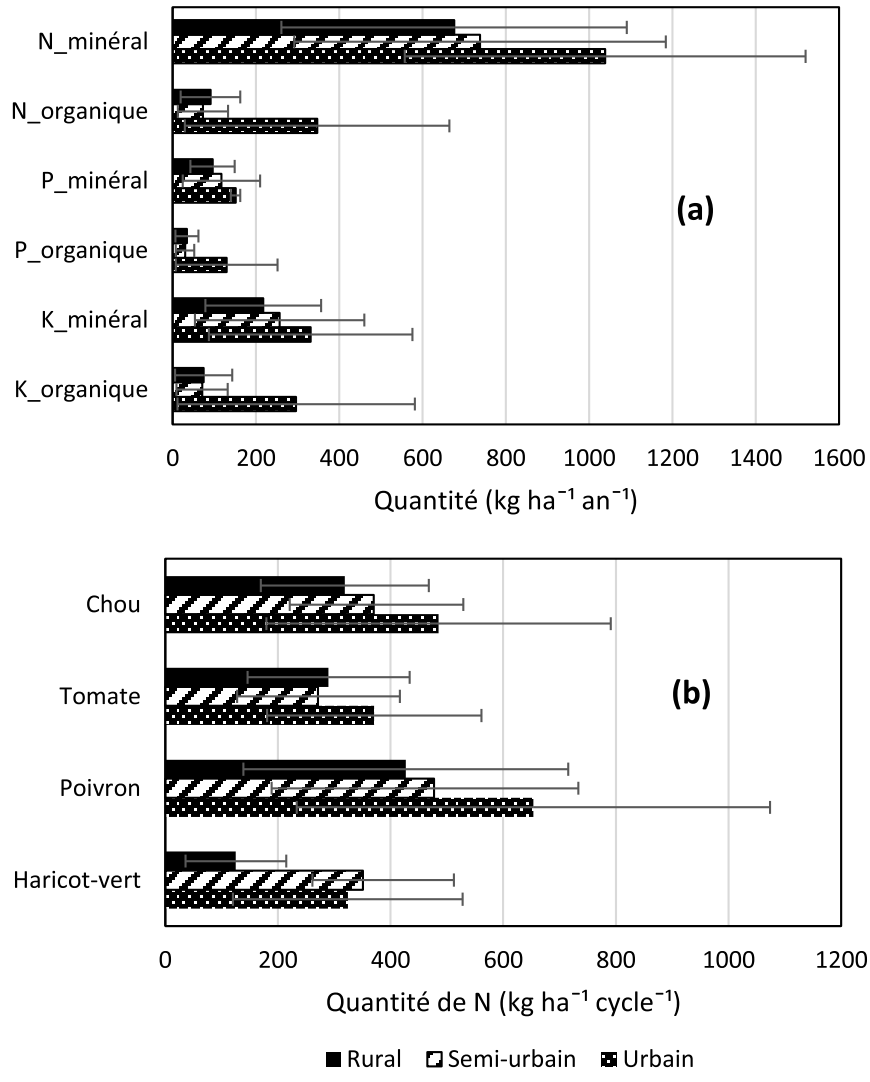

Fig. 3. Quantités annuelles moyennes (et écart-type) de N, P et K apportées par ha de maraîchage sous forme organique ou minérale (a) et quantité de $\mathrm{N}$ apportée par ha et par cycle pour quelques cultures (b) en milieu urbain, semi-urbain et rural.

Fig. 3. Annual quantity of mineral or organic N, $P$ and $K$ applied (a) and quantity of $N$ applied per crop (b) in urban, semi-urban and rural areas.

d'amendement ont été répertoriés (fumier de bovins, porcins, volailles, ovins, déchets urbains et compost) et leur importance relative varie selon les milieux. On note une part plus importante des fientes de volailles dans la fourniture de $\mathrm{P}$ en raison de sa plus grande richesse en $\mathrm{P}$.

La quasi-totalité (98-100 \%) des maraîchers, tous milieux confondus, utilise aussi la fumure minérale et très souvent de manière fractionnée. Les engrais minéraux constituent la source majeure de la fertilisation des cultures maraîchères, quel que soit le type de nutriment principal (N, P, K). Ils représentent en moyenne 75,89 et $89 \%$ des apports totaux de $\mathrm{N}$ en milieux urbain, semi-urbain et rural, respectivement; 54 , 76 et $72 \%$ pour le P, et 53,78 et $74 \%$ pour le K (Fig. 4).

$\mathrm{Au}$ total, 35, 29 et 43 préparations commerciales de pesticides ont été recensées en milieu urbain, semi-urbain et rural, respectivement. Seuls 40 à $45 \%$ des pesticides rencontrés sont homologués pour le maraîchage. Les herbicides à base de glyphosate et paraquat sont plus particulièrement utilisés en milieux semi-urbain $(58 \%)$ et rural $(66 \%)$, mais faiblement utilisés en milieu urbain $(18 \%)$ car les superficies exploitées y sont plus faibles. Tous les producteurs utilisent les insecticides et acaricides. Les formulations comme le Lambda super (lambda-cyhalothrine 
(a)

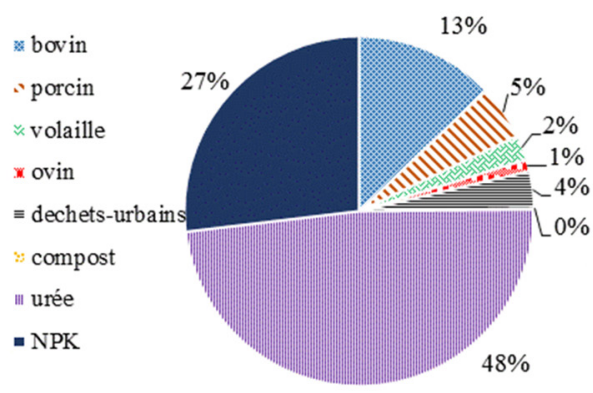

(e)

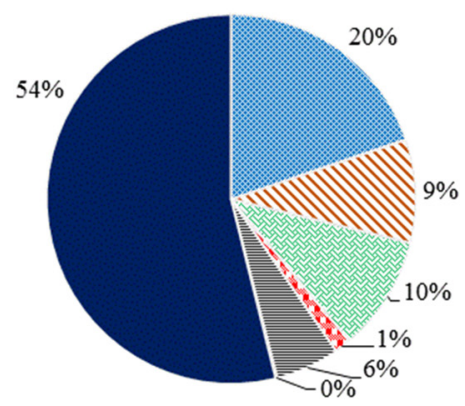

(h)

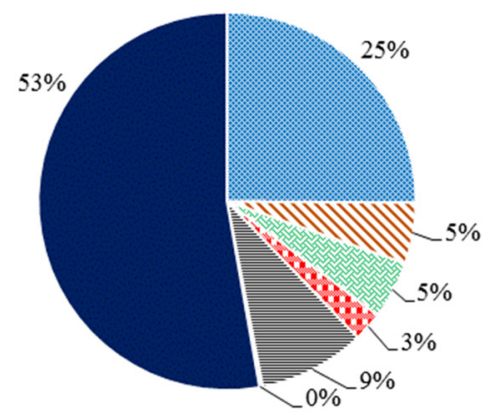

(b)

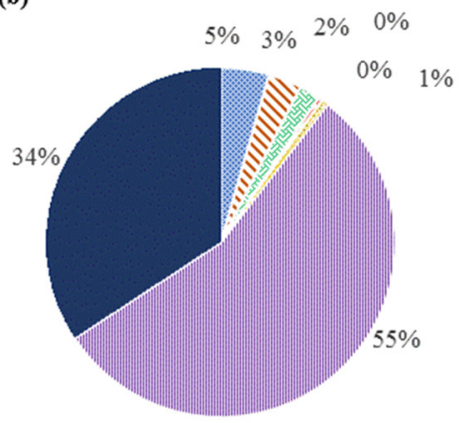

(f)

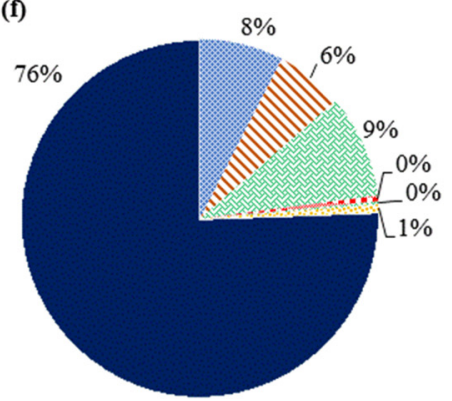

(i)

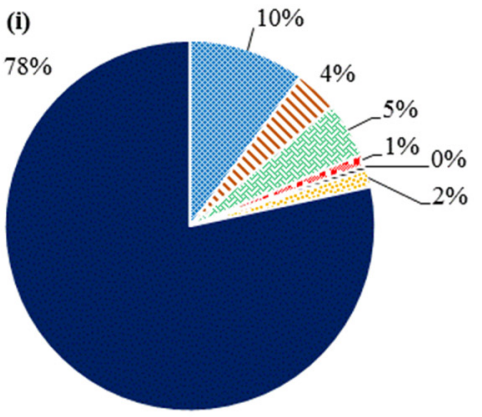

(c)
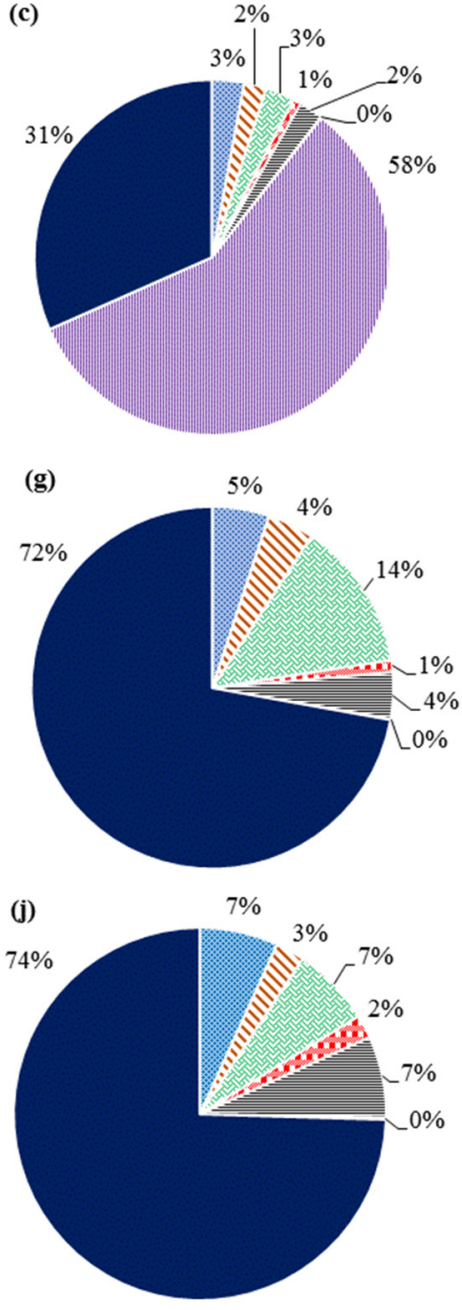

Fig. 4. Proportions de N (a, b, c), P (e, f, g) et K (h, i, j) totaux apportées sous différentes formes en milieu urbain (a, e, h), semi-urbain (b, f, i) et rural (c, g, j).

Fig. 4. Proportion of total $N(a, b, c), P(e, f, g)$ and $K(h, i, j)$ applied in urban $(a, e, h)$, semi-urban $(b, f, i)$ and rural $(c, g, j)$ areas.

$25 \mathrm{~g} / \mathrm{L} ; 71 \%$ des maraîchers), Tihan (flubendiamide $100 \mathrm{~g}$ + spirotetramite $75 \mathrm{~g} / \mathrm{L} ; 50 \%$ ), Avaunt (indoxacarb $150 \mathrm{~g} / \mathrm{L}$; $40 \%$ ), Pacha (acetamipride $10 \mathrm{~g} / \mathrm{L}+$ lambda-cyhalothrine $15 \mathrm{~g} / \mathrm{L} ; 26 \%$ ), CAPT 96 (acetamipride + cyperméthrine ; $26 \%$ ) et Bomec (abamectine $18 \mathrm{~g} / \mathrm{L} ; 24 \%$ ) sont les plus utilisées. Les quantités de matières actives apportées sont les plus élevées sur le poivron, le chou, la tomate et l'aubergine, soit parce qu'ils sont très attaqués (cas du choux) soit parce qu'ils contribuent fortement au revenu des maraîchers. Selon le milieu, 60 à $73 \%$ des producteurs affirment faire le choix des doses selon leur expérience.

Rares sont les producteurs (1 à $2 \%$ ) qui recourent aux pesticides biologiques achetés en ville ou produits par euxmêmes (par exemple, des extraits de feuilles et graines de neem (Azadirachta indica). Près de la moitié des producteurs affirment ne pas avoir d'information sur ces types de pesticides, et ce, malgré le nombre élevé de projets de développement proposant depuis plus d'une décennie ce type d'intrants. En milieu semi-urbain, $42 \%$ des producteurs pensent que les pesticides biologiques sont moins efficaces que les pesticides chimiques, contre $20 \%$ en milieu urbain et $27 \%$ en milieu rural. Les autres évoquent la non disponibilité des produits $(31,6$ et $17 \%$ respectivement en milieu urbain, semiurbain et rural).

\subsection{Perception des producteurs de l'impact de leurs pratiques}

Globalement, la perception de l'impact néfaste des apports de pesticides et d'engrais croît quand on va du milieu urbain au milieu rural (Tab. 2). En ce qui concerne les pesticides, leurs effets négatifs impacteraient avant tout l'eau de surface $(50 \%)$ et dans une moindre mesure le sol $(33 \%)$. Peu de producteurs $(25 \%)$, surtout en milieux urbain et semi-urbain, perçoivent un danger pour les consommateurs de produits maraîchers. Tous milieux confondus, les producteurs sont deux fois plus nombreux à attribuer aux engrais minéraux des impacts négatifs sur la qualité du sol (67\%) qu'aux pesticides (33\%), en affirmant que les engrais «tuent» le sol car ils observent à la longue une perte de 
Tableau 2. Proportion des producteurs percevant un impact négatif de leurs pratiques maraîchères sur le sol, l'eau et les cultures.

Table 2. Proportion of producers perceiving a negative impact of their practices on soil, water and crops.

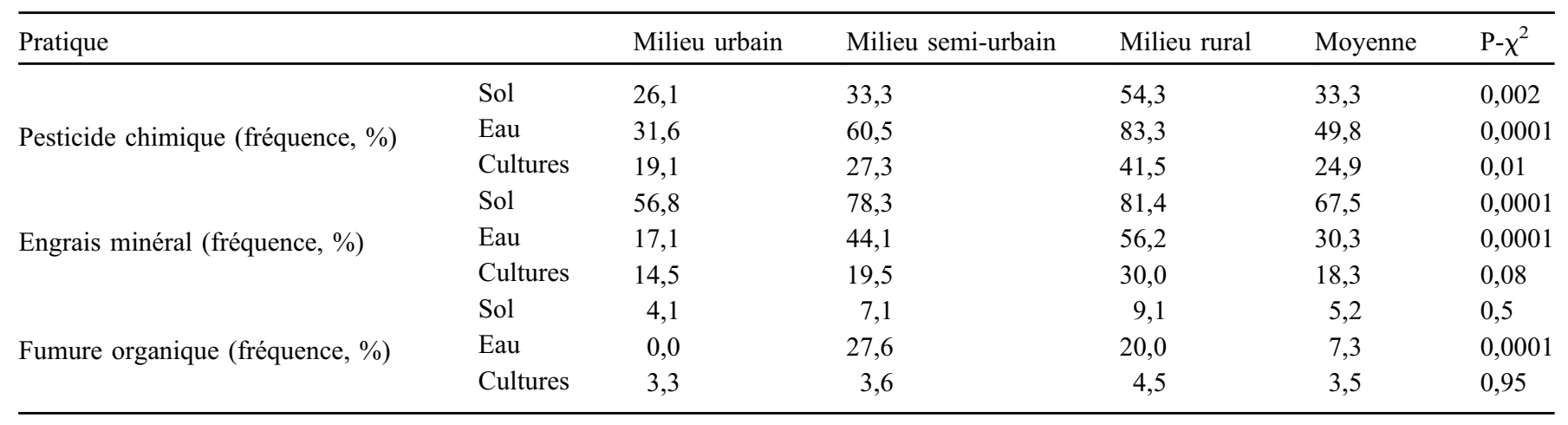

Tableau 3. Caractéristiques des différentes classes d'exploitations basées sur la typologie des pratiques culturales.

Table 3. Characteristics of the different farm classes based on the typology of cropping practices.

\begin{tabular}{|c|c|c|c|c|c|c|c|c|c|c|c|c|c|}
\hline Classe & $\begin{array}{l}\text { Superficie } \\
\left(\mathrm{m}^{2}\right)\end{array}$ & $\begin{array}{l}N \text { total } \\
\left(\mathrm{kg} \mathrm{ha}^{-1}\right. \\
\mathrm{an}^{-1)}\end{array}$ & $\begin{array}{l}\text { Norg/ } \\
\text { Ntot }\end{array}$ & IFT & $\begin{array}{l}\text { Nombre de } \\
\text { traitements/ } \\
\text { spéculation }\end{array}$ & $\begin{array}{l}\text { Nombre de } \\
\text { spéculations }\end{array}$ & $\begin{array}{c}\text { Irrigation } \\
\text { par rivière } \\
(\%)\end{array}$ & $\begin{array}{c}\text { Irrigation } \\
\text { par puits } \\
(\%)\end{array}$ & $\begin{array}{c}\text { Irrigation } \\
\text { manuelle } \\
\text { (arrosoir) } \\
(\%)\end{array}$ & $\begin{array}{c}\text { Irrigation } \\
\text { gravitaire } \\
\text { (moto-pompe) } \\
(\%)\end{array}$ & $\begin{array}{c}\text { Milieu } \\
\text { urbain } \\
(\%)\end{array}$ & $\begin{array}{l}\text { Milieu } \\
\text { semi- } \\
\text { urbain } \\
(\%)\end{array}$ & $\begin{array}{c}\text { Milieu } \\
\text { rural } \\
(\%)\end{array}$ \\
\hline $\begin{array}{l}\text { Très intensive } \\
(n=76)\end{array}$ & 811 & 1828 & 0,25 & 65 & 7 & 5 & 46 & 54 & 96 & 4 & 84 & 15 & 1 \\
\hline Intensive $(n=50)$ & 1119 & 981 & 0,21 & 42 & 5 & 5 & 67 & 33 & 67 & 33 & 56 & 27 & 17 \\
\hline $\begin{array}{l}\text { Peu intensive } \\
(n=127)\end{array}$ & 7829 & 671 & 0,12 & 23 & 9 & 5 & 70 & 30 & 10 & 90 & 25 & 18 & 57 \\
\hline
\end{tabular}

Norg/Ntot : rapport azote organique / azote total; IFT: indice de fréquence de traitements phytosanitaires.

fertilité des sols recevant des engrais minéraux. Une minorité de producteurs en milieu urbain et environ la moitié en milieux semi-urbain et rural affirment que les engrais ont un effet néfaste sur l'eau, qui, selon eux, changent son goût.

Quant à la fumure organique, la quasi-totalité des producteurs affirme qu'elle a un effet bénéfique sur le sol et les cultures maraîchères. Tous les producteurs urbains pensent que la fumure organique est sans effet sur la qualité de l'eau des nappes et de surface. Par contre, 28 et $20 \%$ des producteurs semi-urbains et ruraux pensent que le fumier, utilisé à forte dose, peut polluer l'eau des rivières.

\subsection{Typologie des producteurs en fonction des pratiques}

La CAH a permis de distinguer 4 groupes d'exploitants (Tab. 3). La classe 1 comporte $25 \%$ de l'échantillon. Elle regroupe principalement les exploitations de très petite taille $\left(811 \mathrm{~m}^{2}\right)$ mais «très intensives» car on y observe les taux d'application de $\mathrm{N}$ et les IFT les plus élevés. La part de l'azote organique dans l'azote total y est cependant la plus élevée. La quasi-totalité des exploitations de cette classe se situe en milieu urbain (84\%), avec pour principal mode d'irrigation l'arrosage manuel avec des arrosoirs (96\%).

La classe 2, nommée «classe intensive» (17\% de l'échantillon total), est constituée d'exploitations dont la superficie moyenne est de $1119 \mathrm{~m}^{2}$, situées en majorité en milieu urbain (56\%). La quantité d'azote apportée est réduite de moitié et l'IFT d'un tiers environ par rapport à la classe «très intensive». Environ un cinquième de l'azote $\mathrm{y}$ est apporté sous forme organique et l'arrosage manuel y est dominant $(67 \%)$.

La classe 3, appelée «moyennement intensive » $(16 \%$ des exploitations), regroupe les exploitations ayant des superficies maraîchères moyennement grandes $\left(2463 \mathrm{~m}^{2}\right)$, situées en majorité en milieu rural (52\%). L'apport de $\mathrm{N}$ total et la part de l'azote organique est comparable à la classe "très intensive", mais l'IFT est $38 \%$ plus faible. L'irrigation est gravitaire dans $57 \%$ des cas et utilise l'eau pompée à partir des rivières.

Enfin la classe 4, nommée «peu intensive» $(42 \%$ des exploitations), regroupe les plus grandes exploitations $\left(7829 \mathrm{~m}^{2}\right)$ qui se trouvent majoritairement en milieu rural. L'IFT, les doses d'azote, mais aussi la part de l'azote organique y sont les plus faibles. L'irrigation gravitaire à partir d'eau de rivière pompée est largement dominante $(90 \%$ des exploitations de cette classe). 
Ces différentes classes différencient bien les systèmes maraîchers urbains «intensifs à très intensifs » des systèmes maraîchers ruraux «moyennement à peu intensifs ». En milieu semi-urbain, les exploitations se répartissent de façon relativement homogène entre les 4 classes, avec cependant une prédominance des exploitations «intensives à moyennement intensives $»$.

\section{Discussion}

\subsection{Caractéristiques générales}

Les superficies cultivées annuellement en maraîchage par exploitation sont en moyenne 3 fois plus faibles en milieu urbain qu'en milieu rural. En milieu urbain, l'exiguïté des parcelles est due au morcellement de la propriété transmise par héritage, à la recherche de terres par des personnes sans emploi en ville pour se lancer dans le maraîchage afin de subvenir à leurs besoins, et surtout à la pression de l'urbanisation qui réduit inexorablement la surface cultivable en ville. Cette limitation de l'espace cultivable entraîne les producteurs vers une stratégie d'intensification qui vise à produire beaucoup sur de petites surfaces, en privilégiant des pratiques habituelles dans ce cas : utilisation à fortes doses de fumures organiques et minérales et de pesticides, associations culturales plus fréquentes. Cette intensification est facilitée en milieu urbain par la proximité des commerçants d'intrants et des marchés, en particulier pour écouler très rapidement les légumes feuilles caractéristiques des associations de cultures. Par ailleurs, Robineau (2018) a noté la présence de nombreux éleveurs dans la ville de Bobo-Dioulasso, qui maintiennent des relations d'interdépendance avec les maraîchers urbains pour la fourniture de fumier de bovins et de porcs. Les déchets urbains (ordures ménagères), très partiellement collectées par la municipalité, sont laissés au bord des rues ou le long des ruisseaux et deviennent également une ressource en fumure organique pour les maraîchers urbains (Robineau et Dugué, 2018). La qualité de ces déchets présente cependant un enjeu particulier en termes sanitaires, du fait de la présence possible d'éléments traces métalliques (Smith et al., 2004), de germes pathogènes pour les humains et peut-être de résidus d'antibiotiques. Des actions de promotion d'amendements organiques de qualité, indemnes d'éléments nocifs, sont à encourager en milieu urbain. Pour cela, il est nécessaire de réaliser en amont une étude de faisabilité afin d'anticiper les éventuels obstacles, tels que le coût de la collecte des matières organiques premières et le tri sélectif des ordures ménagères, et la mise en place de filières de production de compost à partir de déchets ménagers et d'agro-industries.

\subsection{Doses de fertilisant}

La majorité des producteurs combinent fertilisation organique et minérale, comme cela a été observé ailleurs (Ahouangninou, 2013; Abdulkadir et al., 2013), mais les apports minéraux sont largement dominants, surtout pour l'azote. Les apports d'azote varient de 125 à $654 \mathrm{~kg} \mathrm{~N} \mathrm{ha}^{-1}$ par cycle de production en fonction des cultures et de la zone (Fig. 3b). Pourtant, selon D’Arondel de Hayes et Traoré (1990), la dose nécessaire pour les légumes en zone soudano-sahélienne varie de 70 à $140 \mathrm{~kg} \mathrm{~N} \mathrm{ha}^{-1}$ selon les cultures. Sangaré (2012), après avoir noté des doses d'azote pouvant atteindre $800 \mathrm{~kg} \mathrm{~N}$ $\mathrm{ha}^{-1}$ par cycle, a expliqué ces apports largement excédentaires par une méconnaissance des maraîchers de la valeur fertilisante des divers fertilisants et des besoins des cultures. Cet excès d'apport d'azote est préjudiciable à la qualité des eaux souterraines et des cours d'eau et pourrait entraîner des déséquilibres alimentaires pour les consommateurs de légumes feuilles. Il serait donc nécessaire d'élaborer une méthode d'apport raisonnée des fertilisants pour les principales cultures maraîchères et rotations, qui soit accessibles aux maraîchers généralement non alphabétisés. Pour cela, il conviendrait de mieux quantifier les besoins des cultures et rotations annuelles les plus courantes, ainsi que la valeur fertilisante des amendements afin de rationaliser les apports (fractionnés, localisés), en priorité pour les exploitations des classes «très intensive », « intensive» et « moyennement intensive».

La part des engrais minéraux dans les apports totaux de nutriments $(\mathrm{N}, \mathrm{P}, \mathrm{K})$ est plus élevée en milieu rural, malgré une plus grande sensibilité des producteurs de ce milieu aux impacts négatifs de ce type d'engrais sur l'environnement. Ceci pourrait s'expliquer d'abord par des contraintes de disponibilités de la fumure organique pour le maraîchage. En milieu rural, il est certes plus facile de faire de l'élevage qu'en ville, mais il faut considérer d'une part que les maraîchers ne possèdent pas tous des animaux d'élevage et, d'autre part, qu'une culture alimentaire de base comme le maïs pluvial a très souvent la priorité en termes d'utilisation de la fumure organique (Vall et al., 2011). Par ailleurs, les exploitations rurales peuvent manquer de fumure organique et de moyens pour la transporter, ainsi que de main-d'œuvre nécessaire à son application au vu des plus grandes surfaces cultivées en milieu rural (jusqu'à 0,78 ha pour la classe «peu intensive»). Particulièrement pour les exploitations de classe «peu intensive», afin de contribuer à améliorer la durabilité des systèmes maraîchers, il conviendrait de sensibiliser les maraîchers à l'intérêt d'accroitre la part des amendements organiques, afin d'aboutir à une combinaison équilibrée entre fumures organiques et minérales. Pour cela, et quel que soit le milieu, il conviendrait de développer un élevage intégré aux systèmes maraîchers et de promouvoir le compostage des ordures ménagères et des résidus de cultures.

\subsection{Utilisation des pesticides}

Tous les producteurs utilisent les pesticides chimiques, qui sont non homologués pour plus de la moitié d'entre eux, tels que déjà rapporté par Son et al. (2017). Parmi les formulations les plus fréquentes, l'Avaunt et le CAPT 96 sont destinés au coton et non aux cultures maraîchères. Le Lamda super (lambda-cyhalothrine $25 \mathrm{~g} / \mathrm{L}$ ), qui est pourtant utilisé par la majorité des maraîchers, n'est pas autorisé par le conseil sahélien des pesticides. Cette substance active ainsi que le paraquat sont interdits par la Commission européenne (base de donnée de la Commission européenne), compte tenu de leur toxicité. En recourant aux pesticides chimiques de synthèse, les producteurs visent à garantir l'aspect physique et la rentabilité des cultures dont les coûts de production sont élevés (Ahouangninou, 2013). Le faible niveau d'instruction des producteurs occasionne une méconnaissance des principaux 
déprédateurs des cultures et des difficultés de lecture des modalités d'utilisation des pesticides figurant sur les emballages, engendrant ainsi une utilisation excessive et parfois inadaptée des produits phytosanitaires (Son et al., 2017). De plus, la mauvaise connaissance des surfaces cultivées rend le calcul des doses difficile pour ceux qui sont non alphabétisés.

De manière générale, il est impératif de réduire la pression des pesticides chimiques dans toutes les classes. Cependant, une attention particulière doit être portée aux producteurs des classes «très intensive » et «intensive » en milieu urbain, qui ne semblent pas sensibles aux impacts des pesticides sur l'environnement et à la qualité sanitaire de leur production, compte tenu du fait que la plupart des projets de sensibilisation sont plutôt orientés vers le milieu rural. Les biopesticides étant très mal connus par les producteurs malgré l'intervention de certains projets, le développement et la promotion de ces biopesticides (par exemple à travers la mise en place de petites unités locales de production d'extraits et d'huiles essentielles de plantes), ainsi que la lutte biologique (utilisation de Trichoderma harzianum, par exemple, pour réduire la pression fongique tellurique; Dabire et al., 2016), seraient des actions essentielles en vue de réduire l'usage des insecticides et fongicides chimiques. Enfin, une sensibilisation des consommateurs pourrait inciter les autorités publiques à faire appliquer les lois en vigueur règlementant l'usage des pesticides et conduire les producteurs à modifier leurs pratiques, permettant à ces derniers une meilleure valorisation économique de leurs produits maraîchers issus de systèmes de culture agroécologiques.

En milieu rural, le fort taux d'utilisation d'herbicides totaux sur les parcelles maraîchères est surtout lié aux grandes superficies des parcelles et vise à réduire la charge de travail liée au sarclage. Une mécanisation des opérations de sarclage, ainsi que la pratique de l'irrigation localisée comme le goutteà-goutte, permettraient de réduire l'usage des herbicides dans les exploitations des classes «moyennement intensive» et «peu intensive».

\subsection{Mode d'irrigation et système de culture}

En milieu rural (exploitations des classes «moyennement intensive » et « peu intensive»), pour diminuer la pénibilité du travail et le besoin en main-d'œuvre qui reste souvent très couteuse dans ce milieu (Ahouangninou, 2013), l'irrigation se fait le plus souvent à la raie avec des motopompes, car les surfaces maraîchères y sont relativement importantes (respectivement 0,25 ha et $0,78 \mathrm{ha}$ ). La part des eaux de rivière dans l'irrigation croît de la classe «très intensive» à la classe «peu intensive ». Vu la qualité sanitaire souvent moindre des eaux de surface en milieu urbain, liée à des pollutions diverses (métaux lourds ; bactéries) (Abdu et al., 2011; Diogo et al., 2010), une sensibilisation particulière mérite d'être menée pour les producteurs concernés en complément d'une meilleure gestion des eaux usées.

Sur l'ensemble des sites, aucun producteur n'a mis en œuvre des pratiques relevant exclusivement de l'agriculture biologique ou agroécologique au sens global du terme. La pratique des rotations et des associations de cultures semble cependant bien ancrée chez une majorité de producteurs, formant ainsi une base de travail positive pour faire évoluer le maraîchage vers plus de durabilité. Cependant, l'objectif premier de ces associations et rotations n'étant pas de lutter contre les ravageurs, mais plutôt de pallier le manque de terre et de répondre à des raisons économiques, un meilleur encadrement des producteurs pour une amélioration des associations et rotations avec l'usage de plantes répulsives pourrait contribuer à une réduction des quantités de pesticides utilisés.

\section{Conclusion}

Malgré le recours généralisé à des pesticides de synthèse et à la fertilisation minérale par toutes les classes de producteurs, mais particulièrement intensif en milieu urbain, les pratiques de la rotation et des associations culturales ainsi que la fertilisation organique mises en œuvre par la majorité des producteurs constituent un atout pour initier la transition agroécologique des systèmes de culture maraîchers. La typologie des maraîchers basée sur les pratiques de production a permis de définir quatre types d'exploitations présentant des défis spécifiques à relever pour réussir cette transition agroécologique. Le premier défi commun à tous les types de maraîchers réside dans le développement de méthodes efficaces de gestion intégrée des ravageurs. Le second défi plus spécifique du maraîchage urbain est la mise en œuvre d'une gestion raisonnée de la fertilisation des cultures maraîchères par une réduction des doses d'engrais, surtout de l'azote. De façon générale, la sensibilisation aux impacts sanitaires et environnementaux des pratiques culturales maraîchères doit être renforcée, tout particulièrement en milieu urbain, auprès des producteurs mais peut-être surtout auprès des consommateurs, à travers qui pourra émerger une demande pour des produits issus de systèmes de culture plus respectueux de la santé et de l'environnement. Une évaluation socio-économique des freins à l'adoption des pratiques agroécologiques permettra de mener des actions plus efficaces.

Remerciements. Ce travail a bénéficié du soutien de l'Académie de recherche et d'enseignement supérieur (ARES-CDD) à travers le projet de recherche pour le développement «PARticipative Agroecological DEvelopment (PARADE)».

\section{Références}

Abdu N, Abdulkadir A, Agbenin JO, Buerkert A. 2011. Vertical distribution of heavy metals in wastewater-irrigated vegetable garden soils of three West African cities. Nutr Cycl Agroecosyst 89: 387-397. DOI: 10.1007/s10705-010-9403-3.

Abdulkadir A, Leffelaar PA, Agbenin JO, Giller KE. 2013. Nutrient flows and balances in urban and peri-urban agroecosystems of Kano, Nigeria. Nutr Cycl Agroecosyst 95: 231-254. DOI: 10.1007/ s10705-013-9560-2.

Ahouangninou CCA. 2013. Durabilité de la production maraîchère au sud-Bénin : un essai de l'approche écosystémique. Thèse de Doctorat Unique. Bénin: Université d'Abomey-Calavi, 344 p. https://agritrop.cirad.fr/572410/1/document_572410.pdf.

Dabire TG, Bonzi S, Somda I, Legreve A. 2016. Évaluation in vitro de l'activité antagoniste d'isolats de Trichoderma harzianum Pers. contre trois espèces fongiques pathogènes de l'oignon au Burkina Faso. Tropicultura 34(3): 313-322. 
D’Arondel de Hayes J, Traoré G. 1990. Cultures maraîchères en zone Soudano-Sahélienne. Recueil de fiches techniques, 79 p. Montpellier, France: CIRAD-IRAT, INERA. http://agritrop.cirad.fr/ 341076/1/ID341076.pdf.

Diogo RVC, Buerkert A, Schlecht E. 2010. Horizontal nutrient fluxes and food safety in urban and peri-urban vegetable and millet cultivation of Niamey, Niger. Nutrient Cycling in Agroecosystems 87: 81-102.

Dossa LH, Abdulkadir A, Amadou H, Sangaré S, Schlecht E. 2011. Exploring the diversity of urban and peri-urban agricultural systems in Sudano-Sahelian West Africa: an attempt towards a regional typology. Landscape and Urban Planning 10(2): 196206. DOI: 10.1016/j.landurbplan.2011.04.005.

Gafsi M, Dugué P, Jamin JY, Brossier J. 2007. Exploitations agricoles d'Afrique de l'Ouest et du Centre. Versailles, France: Quae, 472 p. https://cgspace.cgiar.org/bitstream/handle/10568/100821/ 1395 PDF.pdf? sequence $=1$.

Landais E, Deffontaines JP, Benoît M. 2016. Les pratiques des agriculteurs. Point de vue sur un courant nouveau de la recherche agronomique. Études rurales 109: 125-158. DOI: 10.3406/ rural.1988.3226.

Robineau O. 2018. Elever des porcs dans une ville d'Afrique de l'Ouest : arrangements entre acteurs pour gérer la proximité villeélevage. Revue d'Elevage et de Médecine Vétérinaire des Pays Tropicaux 71(1-2) (dossier L'élevage porcin dans les pays tropicaux): 10 p. DOI: 10.19182/remvt.31288.

Robineau O, Dugué P. 2018. A socio-geographical approach to the diversity of urban agriculture in a West African city. Landscape and Urban Planning 170: 48-58. DOI: 10.1016/j.landurb plan.2017.09.010.
Sangaré SAKSB. 2012. Water and nutrient use efficiency and the vertical leaching losses in urban vegetable cropping systems in Bobo-Dioulasso (Burkina Faso). Thèse de doctorat. Belgique: Université Catholique de Louvain, 193 p. https://dial.uclouvain.be/ $\mathrm{pr} /$ boreal/object/boreal\%3A110879/datastream/PDF 01/view.

Smith OB, Moustier P, Mougeot LJA, Fall A. 2004. Développement durable de l'agriculture urbaine en Afrique francophone. Enjeux, concepts et méthodes. Montpellier, France: CIRAD/CRDI, 176 p.

Son D, Somda I, Legrève A, Schiffers B. 2017. Pratiques phytosanitaires des producteurs de tomates du Burkina Faso et risques pour la santé et l'environnement. Cahiers Agricultures 26: 25005. DOI: 10.1051/cagri/2017010.

Stassart PM, Baret P, Grégoire JC, Hance T, Mormont M, Reheul D, et al. 2012. L'agroécologie: trajectoire et potentiel pour une transition vers des systèmes alimentaires durables. Dijon, France: Educagri editions, 21 p. https://orbi.uliege.be/bitstream/2268/ $130063 / 1 /$ Agroecologie $\% 20$ Stassart $\% 20 \% 2 \mathrm{c} \% 20$ Baret $\% 20$ et $\%$ 20al.\%20GIRAF.pdf.

Traoré K, Toé AM. 2008. Capitalisation des initiatives sur les bonnes pratiques agricoles au Burkina Faso. Ministère de l'Agriculture de l'Hydraulique et des Ressources Halieutiques, 99 p. https://www. doc-developpement-durable.org/file/Outils-\&-bonnes-pratiquestravail-de-la-Terre/bonnes-pratiques-agricoles/BonnesPratiquesA gricoles Burkina.pdf.

Vall E, Koutou M, Blanchard M, Coulibaly K, Diallo MA, et al. 2011. Intégration agriculture-élevage et intensification écologique dans les systèmes agrosylvopastoraux de l'Ouest du Burkina Faso, province du Tuy. Partenariat, modélisation, expérimentations : quelles leçons pour la conception de l'innovation et l'intensification écologique? Bobo-Dioulasso, Burkina Faso. 13 p. https://hal. archives-ouvertes.fr/hal-00718613.

Citation de l'article : Ouédraogo RA, Kambiré FC, Kestemont M-P, Bielders CL. 2019. Caractériser la diversité des exploitations maraîchères de la région de Bobo-Dioulasso au Burkina Faso pour faciliter leur transition agroécologique. Cah. Agric. 28: 20. 